\title{
Incidence of contrast-induced nephropathy a prospective study
}

\author{
Sameer Peer*, Naseer Ahmad Choh, Tariq Ahmad Gojwari \\ Department of Radiodiagnosis and Imaging, Sher-i-Kashmir Institute of Medical Sciences, Soura, Srinagar, Jammu and Kashmir, India
}

\section{ART I C L E I N F O}

Article Type:

Original

\section{Article History:}

Received: 9 September 2016

Accepted: 12 November 2016

Published online: 25 November 2016

Keywords:

Contrast agent

Heart failure

Nephropathy

Osmolality

Serum creatinine

Viscosity

\begin{abstract}
A B S T R A C T
Introduction: Contrast-induced acute kidney injury (AKI) is one of the most common causes of hospital-acquired AKI.

Objectives: To determine the incidence of contrast induced nephropathy (CIN), to identify significant risk factors associated with CIN and to compare the variations in serum creatinine levels with and without contrast exposure.

Patients and Methods: A total of 222 patients (124 males and 98 females with mean age of $48.96 \pm 16.74$ years) who received iodinated contrast agents during different procedures over a period of 18 months were included in the study. CIN was defined as a relative increase of $>25 \%$ or an absolute increase of $>0.5 \mathrm{mg} / \mathrm{dL}$ in serum creatinine levels 4 days post- procedure. 148 patients ( 82 males and 66 females with mean age of $47.48 \pm 17.21$ years) who did not receive any contrast agent were included as controls to determine the frequency with which the variations in serum creatinine levels fulfilled the definition of CIN.

Results: The overall incidence of CIN was $12.6 \%$. Incidence of CIN was $32.6 \%$ after percutaneous coronary intervention $(\mathrm{PCI})$ and $7.38 \%$ after IV contrast exposure $(P<0.0001)$. Multivariate logistic regression analysis of risk factors revealed heart failure $(P=0.001)$, pre-procedure serum creatinine level $\geq 1.5 \mathrm{mg} / \mathrm{dL}(P=0.005)$ and nature of contrast agent $(P=0.001)$, as independent risk factors of CIN. $2.02 \%$ patients in control group showed variations in serum creatinine levels within the range corresponding to the definition of CIN. Conclusion: Heart failure, pre-procedural serum creatinine of $\geq 1.5 \mathrm{mg} / \mathrm{dL}$ and the nature of contrast agent are independent predictors of CIN.
\end{abstract}

Implication for health policy/practice/research/medical education:

Contrast induced AKI is one of the most common causes of hospital-acquired AKI. Most of the prospective studies lack a control group for comparison. This study evaluates the epidemiology of CIN and compares the findings with a matched control group. Heart failure, pre-procedure serum creatinine level $\geq 1.5 \mathrm{mg} / \mathrm{dL}$ and nature of contrast medium used can independently predict the likelihood of Contrast-induced nephropathy.

Please cite this paper as: Peer S, Choh NA, Gojwari TA. Incidence of contrast-induced nephropathy a prospective study. J Renal Inj Prev. 2017;6(3):192-198. DOI: 10.15171/jrip.2017.37.

\section{Introduction}

Contrast-induced nephropathy (CIN) is widely recognised as the third most common cause of hospital acquired acute kidney injury (AKI) and accounts for $11 \%-12 \%$ of all cases of in-hospital AKI and an in-hospital mortality rate of $6 \%$ (1-3). CIN occurs after intravascular administration of iodinated contrast media during diagnostic and/or interventional procedures. The risk of development of CIN is highest with coronary angiography and percutaneous coronary intervention (PCI) $(2,4)$. CIN occurs in about $14.5 \%$ of patients after coronary interventions with inhospital mortality rate of $7.1 \%$ in patients without the need for dialysis and $35.7 \%$ in those requiring dialysis (5). $\mathrm{CIN}$ is uncommon in patients with normal baseline renal function. It occurs more frequently in patients with preexisting renal impairment particularly if it is associated with diabetes (6).

CIN is defined as an acute deterioration of renal function after intravascular exposure to contrast media in absence of other causes. The serum creatinine levels begin to rise within 24-48 hours, peak at 2-3 days and return to the baseline values within 2 weeks (7). The most commonly used definition of CIN in the literature is either a relative increase in serum creatinine of $25 \%$ or an absolute 
increase of $0.5 \mathrm{mg} / \mathrm{dL}$ from a baseline value within 48 to 72 hours after contrast exposure. Additionally, there must be no other alternative cause for the elevation of serum creatinine levels and it must persist for 2-5 days $(8,9)$.

Most cases of CIN are self-limiting with serum creatinine levels returning to baseline within 2 weeks $(10,11)$. In more severe cases, a delayed peak and a slow return to a steady state which is above the baseline may be seen. In a small number of cases, renal replacement therapy may be required. Among those who require dialysis, median 2 -year survival is estimated to be approximately $19 \%$ with an in-hospital mortality as high as 36\% (12).

The incidence of CIN varies widely among the publications in the literature and most of the prospective studies lack a suitable control group. In this study, we evaluated the epidemiology of CIN with inclusion of a matched control group for comparison with contrast group of patients to study the variations in serum creatinine levels with and without contrast administration.

\section{Objectives}

The objective of our study was to determine the incidence of CIN among patients undergoing various procedures at our institution. We also evaluated various risk factors associated with CIN. We included a control group of patients who did not receive any contrast agent but were comparable to the group of patients who received contrast injection with respect to other risk factors in order to determine the variations in serum creatinine levels in absence of contrast exposure.

\section{Patients and Methods}

This study was conducted in a tertiary care hospital in north India for a period of 18 months.

\section{Patient selection}

This was an observational study which included the patients who underwent intravenous urography (IVU), contrast-enhanced computed tomography (CECT) and PCI. Both inpatients as well as outpatients were included in the study. All the procedures were conducted with all the necessary equipment to manage contrast reactions kept by the side of the patient for use as and when required. All the patients were evaluated as per a proforma which included the demographic details, clinical history and diagnosis. A pre-procedure serum creatinine level was recorded. In cases where the available serum creatinine levels were more than 1 week old, the patients were requested to get a fresh serum creatinine level done before the procedure. Using the modification of diet in renal disease (MDRD) or Schwartz formula (wherever applicable), estimated glomerular filtration rate (eGFR) was calculated. Patients with eGFR $\geq 60 \mathrm{~mL} / \mathrm{min} / 1.73 \mathrm{~m}^{2}$ were classified into high-risk group while those with eGFR $<60 \mathrm{~mL} / \mathrm{min} / 1.73 \mathrm{~m}^{2}$ were classified into low-risk group. In high-risk cases, the patients were given intravenous (IV) hydration and reno-protective measures before proceeding with the contrast study except in emergency cases where the contrast-enhanced procedure was considered necessary despite the high-risk.

During the procedure, documentation of the particular contrast material used, dose of the contrast material and the route of administration was done.

Following the procedure, serum creatinine levels were obtained on the fourth day post-procedure from the same reference laboratory where pre-procedure serum creatinine levels were determined to negate the interlaboratory variability in the measurements of serum creatinine levels. Post-procedure serum creatinine levels thus obtained were compared with the pre-procedure levels to determine whether CIN had occurred or not.

\section{Definition of contrast-induced nephropathy}

For the purpose of this study, CIN was defined as:

1. Relative rise of serum creatinine levels by $>25 \%$ of the pre-procedure level

2. An absolute rise in serum creatinine levels by $>0.5 \mathrm{mg} /$ $\mathrm{dL}$ above the baseline

Post-procedure levels obtained 4 days after the administration of iodinated contrast material.

\section{Exclusion criteria}

The following group of patients were excluded from the study:

1. Patients having a history of allergic reaction to contrast agents or iodine.

2. Patients who did not give consent to undergo the procedure involving contrast medium administration.

3. Patients who were on a dialysis regimen.

4. Patients who did not have a pre-procedure serum creatinine level analysis and post-procedure serum creatinine level analysis done at the same reference laboratory.

5. Pregnant females.

\section{Selection of control group}

A group of patients who did not receive any intravascular contrast agents but were otherwise comparable to the group of patients who received iodinated contrast agent was selected as the control group. Both inpatients and outpatients were included in this group. The purpose of selection of the control group was to determine the effect of contrast medium on serum creatinine levels while controlling for other factors which could possibly account for the elevation of the serum creatinine levels. In this group of patients, two serial serum creatinine levels were obtained from the same reference laboratory 4 days apart. The two serum creatinine levels thus obtained were compared to determine whether the variation falls within the definition of CIN or not.

\section{Ethical issues}

The research followed the tenets of the Declaration of Helsinki; informed consent was obtained; and the research 
was approved by the ethical committee of Sher-i-Kashmir Institute of Medical Sciences.

\section{Statistical analysis}

The continuous variables are presented as mean \pm standard deviation (SD) and categorical variables as number and percentage. The association between categorical variables was assessed using chi-square test. Comparison of means was done using $t$ test. Multivariate logistic regression was used to determine the independent variables for prediction of the occurrence CIN. Analysis was performed in Microsoft Excel and SPSS version 16. $P$ value less than 0.05 was considered as statistically significant.

\section{Results}

In this prospective study, 222 patients who received intravascular iodinated contrast media during various procedures were evaluated. CIN occurred in 28 (12.6\%) patients overall. The mean age of patients included in the "contrast group" was $48.96 \pm 16.74$ years and $44.15 \%$ were females.
The various risk factors evaluated in our study were age, gender, body mass index (BMI), type of procedure, route of administration, nature of contrast agent, volume of contrast, hypertension, diabetes mellitus, active malignancy, chemotherapy, pre-procedural serum creatinine levels, previous contrast exposure within one year, coronary artery disease, peripheral arterial disease, multiple myeloma, hypotension (systolic blood pressure $<90 \mathrm{~mm} \mathrm{Hg}$ ), heart failure, high-risk (eGFR $<60 \mathrm{~mL} /$ $\min / 1.73 \mathrm{~m}^{2}$ ), nephrotoxic medications and inpatient status. A comparative univariate analysis of different risk factors among those who developed CIN and those who did not is summarized in Table 1 .

Age, BMI, type of procedure, route of administration, nature of contrast agent, volume of contrast, hypertension, diabetes mellitus, pre-procedural serum creatinine levels, coronary artery disease, hypotension (systolic blood pressure $<90 \mathrm{~mm} \mathrm{Hg}$ ), heart failure, high-risk (eGFR $<60$ $\mathrm{mL} / \mathrm{min} / 1.73 \mathrm{~m}^{2}$ ), nephrotoxic medications and inpatient status were found to be significantly associated with CIN. These risk factors were analysed using multivariate

Table 1. Comparative analysis of various risk factors and clinical characteristics between CIN and No CIN groups

\begin{tabular}{|c|c|c|c|}
\hline Risk factor & $\mathrm{CIN}(\mathrm{n}=28)$ & No CIN (n = 194) & $P$ value \\
\hline Mean age (years) & $59.46 \pm 11.33$ & $47.44 \pm 16.87$ & 0.0003 \\
\hline Female sex & $12(42.85 \%)$ & $86(44.32 \%)$ & 0.88 \\
\hline Mean BMI $\left(\mathrm{kg} / \mathrm{m}^{2}\right)$ & $26.09 \pm 3.61$ & $24.57 \pm 3.76$ & 0.04 \\
\hline Procedure & & & $<0.0001$ \\
\hline IVU $(n=69)$ & $0(0 \%)$ & 69 (100\%) & \\
\hline CECT $(n=107)$ & $13(12.14 \%)$ & $94(87.86 \%)$ & \\
\hline $\mathrm{PCl}(\mathrm{n}=46)$ & $15(32.6 \%)$ & $31(67.4 \%)$ & \\
\hline Route & & & $<0.0001$ \\
\hline $\mathrm{IA}(\mathrm{n}=46)$ & $15(32.6 \%)$ & $31(69.4 \%)$ & \\
\hline $\operatorname{IV}(n=176)$ & $13(7.38 \%)$ & $163(92.62 \%)$ & \\
\hline Nature of contrast agent & & & $<0.0001$ \\
\hline $\operatorname{LOCM}(n=213)$ & $23(10.79 \%)$ & $190(89.20 \%)$ & \\
\hline $\operatorname{IOCM}(n=9)$ & $5(55.55 \%)$ & $4(44.45 \%)$ & \\
\hline Mean volume of contrast agent $(\mathrm{mL})$ & $115.71 \pm 45.31$ & $76.15 \pm 32.18$ & $<0.0001$ \\
\hline Hypertension & $22(78.57 \%)$ & 45 (23.19\%) & $<0.0001$ \\
\hline Diabetes mellitus & $21(75 \%)$ & $58(29.89 \%)$ & $<0.0001$ \\
\hline Malignancy & $7(25 \%)$ & $57(29.38 \%)$ & 0.62 \\
\hline Chemotherapy & $6(21.42 \%)$ & $27(13.91 \%)$ & 0.317 \\
\hline Hypotension & $9(32.14 \%)$ & $7(3.60 \%)$ & $<0.0001$ \\
\hline Heart failure & $14(50 \%)$ & $6(3.09 \%)$ & $<0.0001$ \\
\hline Nephrotoxic medications & $11(39.28 \%)$ & $14(7.21 \%)$ & $<0.0001$ \\
\hline Pre-procedure SCr (mg/dL) & $1.32 \pm 0.33$ & $0.91 \pm 0.24$ & $<0.0001$ \\
\hline Pre-procedure $\mathrm{SCr} \geq 1.5 \mathrm{mg} / \mathrm{dL}$ & $11(39.28 \%)$ & $5(2.57 \%)$ & $<0.0001$ \\
\hline Diabetics with pre-procedural $\mathrm{SCr} \geq 1.5 \mathrm{mg} / \mathrm{dL}$ & $8(28.57 \%)$ & $3(1.54 \%)$ & $<0.0001$ \\
\hline Post-procedure SCr (mg/dL) & $2.53 \pm 1.74$ & $0.98 \pm 0.28$ & $<0.0001$ \\
\hline Coronary artery disease & $19(67.85 \%)$ & $30(15.46 \%)$ & $<0.0001$ \\
\hline Peripheral arterial disease & $3(1.07 \%)$ & $7(3.60 \%)$ & 0.13 \\
\hline Multiple myeloma & $1(3.57 \%)$ & $2(1.03 \%)$ & 0.34 \\
\hline Metformin & $4(14.28 \%)$ & $17(8.76 \%)$ & 0.37 \\
\hline Inpatients & $21(75 \%)$ & $56(28.86 \%)$ & $<0.0001$ \\
\hline High-risk (eGFR <60 mL/min/1.73 m²) & $19(67.85 \%)$ & $34(17.52 \%)$ & $<0.0001$ \\
\hline Previous contrast exposure & $2(7.14 \%)$ & $5(2.57 \%)$ & 0.25 \\
\hline
\end{tabular}

Abbreviations: CIN, contrast-induced nephropathy; IVU, intravenous urography; CECT, contrast-enhanced computed tomography; PCI, percutaneous coronary intervention; IA, intra-arterial; IV, intravenous; LOCM, low-osmolar contrast media; IOCM; iso-osmolar contrast media; SCr, serum creatinine; eGFR, estimated glomerular filtration rate. 
Table 2. Effect of prophylactic measures on incidence of CIN in high-risk patients and those with pre-existing renal insufficiency (preprocedure $\mathrm{SCr} \geq 1.5 \mathrm{mg} / \mathrm{dL}$ ).

\begin{tabular}{lccc}
\hline & CIN & No CIN & P value \\
\hline High-risk $(n=53)$ & & & \\
NS & $13(68.42 \%)$ & $22(64.70 \%)$ & 0.78 \\
NAC & $9(47.36 \%)$ & $6(17.64 \%)$ & 0.02 \\
Bicarbonate & $7(36.84 \%)$ & $6(17.64 \%)$ & 0.12 \\
Renal insufficiency $(n=16)$ & & & \\
NS & $9(81.81 \%)$ & $5(100 \%)$ & 0.32 \\
NAC & $9(81.81 \%)$ & $5(100 \%)$ & 0.32 \\
Bicarbonate & $8(72.72 \%)$ & $3(60 \%)$ & 0.62 \\
\hline
\end{tabular}

Abbreviations: $\mathrm{CIN}$, contrast-induced nephropathy; $\mathrm{SCr}$, serum creatinine; NS, normal saline; NAC, N-acetyl cysteine..

logistic regression. Heart failure $(\beta=4.25, P=0.01)$, preprocedure serum creatinine level $\geq 1.5 \mathrm{mg} / \mathrm{dL}(\beta=3.99$, $P=0.05)$ and nature of contrast material $(\beta=-1.41$, $P=0.01)$ were found to be independent predictors of CIN in our study. Around 83 patients received IV hydration prophylaxis with normal saline while $\mathrm{N}$-acetyl cysteine and IV sodium bicarbonate were received by 17 patients and 14 patients, respectively. The mean pre-procedure serum creatinine levels among those who received normal saline, $\mathrm{N}$-acetyl cysteine and sodium bicarbonate were $1.06 \pm 0.34 \mathrm{mg} / \mathrm{dL}, 1.56 \pm 0.16 \mathrm{mg} / \mathrm{dL}$ and $1.57 \pm 0.16 \mathrm{mg} /$ $\mathrm{dL}$, respectively, and were significantly higher than those who did not receive any of these prophylactic measures. The prophylactic measures did not reduce the incidence of CIN among those with pre-existing renal insufficiency (pre-procedure serum creatinine $\geq 1.5 \mathrm{mg} / \mathrm{dL}$ ) and those in high-risk group (eGFR $<60 \mathrm{~mL} / \mathrm{min} / 1.73 \mathrm{~m}^{2}$ ) as is shown in Table 2.

In the control group $(n=148), 3$ patients had variations in serum creatinine levels, obtained 4 days apart, which fulfilled the definition of CIN used for contrast group. This difference was statistically significant $(2.02 \%$ [control] as compared to $12.6 \%$ [contrast], $P=0.0003$ ). There was significant difference between the mean serum creatinine level obtained on fourth day post-procedure in contrast group and the mean serum creatinine level on fourth day relative to the initial measurement in control group $(1.17 \pm 0.84$ [contrast] as compared to $0.96 \pm 0.41$ [control], $P=0.0050$ ). The groups were matched for other confounding risk factors. Comparison between contrast group and control group is shown in Table 3.

Among those patients who developed CIN postprocedure, 5 required renal replacement therapy (17.85\%) and 4 patients died (14.28\%) in hospital, although CIN as a cause of death could not be established in any patient.

\section{Discussion}

The overall incidence of CIN in our study was $12.6 \%$. The incidence of CIN quoted in the literature varies widely. In studies which included patients who received contrast media only intravenously, the incidence of CIN ranged from $2.5 \%$ to $12 \%(13-16)$. An incidence of $7 \%-50 \%$ has been reported in studies which combined intravenous and intra-arterial contrast media administration $(12,17)$. It appears that the occurrence of nephropathy after contrast exposure is multifactorial and the incidence of CIN is expected to vary among different studies depending on the study design, the population characteristics and the nature of the contrast procedures included in the study among many other possible reasons $(6,18)$. We evaluated three procedures during the course of our study, IVU, contrast enhanced CT and PCI. The incidence of CIN among each of these three procedures was $0 \%, 12.14 \%$

Table 3. Comparison between control group and contrast group

\begin{tabular}{|c|c|c|c|}
\hline Variable & Contrast group $(n=222$ ) & Control group $(n=148)$ & $P$ value \\
\hline Age (mean years) & $48.97 \pm 16.74$ & $47.48 \pm 17.21$ & 0.4106 \\
\hline Males (\%) & 55.85 & 55.40 & 0.9321 \\
\hline $\mathrm{BMI}\left(\right.$ mean $\mathrm{kg} / \mathrm{m}^{2}$ ) & $24.76 \pm 3.76$ & $24.29 \pm 3.02$ & 0.2044 \\
\hline Diabetes (\%) & 35.58 & 34.45 & 0.8237 \\
\hline Hypertension (\%) & 30.18 & 33.78 & 0.4663 \\
\hline Malignancy (\%) & 28.82 & 25.00 & 0.4197 \\
\hline Chemotherapy (\%) & 14.86 & 11.48 & 0.3521 \\
\hline Metformin (\%) & 9.45 & 8.78 & 0.8272 \\
\hline Hypotension (\%) & 7.2 & 4.05 & 0.2098 \\
\hline $\mathrm{Scr} \geq 1.5 \mathrm{mg} / \mathrm{dL}$ & 7.2 & 6.75 & 0.8683 \\
\hline Nephrotoxic drugs (\%) & 11.26 & 9.45 & 0.5790 \\
\hline Previous exposure (\%) & 3.15 & 3.37 & 0.9069 \\
\hline CAD (\%) & 22.07 & 17.5 & 0.2805 \\
\hline PAD (\%) & 4.5 & 2.7 & 0.3744 \\
\hline Multiple myeloma (\%) & 1.35 & 3.3 & 0.2037 \\
\hline Heart failure (\%) & 9.09 & 8.1 & 0.7409 \\
\hline High-risk (\%) & 23.87 & 22.29 & 0.7248 \\
\hline Day $0 \mathrm{SCr}$ (control)/pre-SCr (contrast) & $0.96 \pm 0.29$ & $0.90 \pm 0.35$ & 0.0738 \\
\hline Day $4 \mathrm{SCr}$ (control)/post-SCr (contrast) & $1.17 \pm 0.84$ & $0.96 \pm 0.41$ & 0.0050 \\
\hline Inpatients (\%) & 34.68 & 34.45 & 0.9637 \\
\hline
\end{tabular}

Abbreviations: BMI, body mass index; SCr, serum creatinine; CAD, coronary artery disease; PAD, peripheral arterial disease. 
and $32.6 \%$ respectively. The reason for this difference could be related not only to different the clinical profile of the patients undergoing these procedures, but also to the dose and route of administration of the contrast material. In general, the patients who underwent IVU were mostly outpatients with lesser co-morbidities and associated risk factors, lesser dose of contrast agent and intravenous route of administration. Intra-arterial (IA) route of administration has been associated with a higher incidence of CIN (12-18). The possible mechanisms responsible for this association could be a higher concentration of the contrast agent at or above the level of renal arteries after IA administration. On the other hand, dilution of contrast may have some protective effect after IV administration. Atheroembolism following IA administration may also be a contributing factor $(6,17)$.

In the literature, there is no consensus regarding the relative nephrotoxicity of low-osmolar contrast media (LOCM) versus iso-osmolar contrast media (IOCM). A lower incidence of CIN with IOCM as compared with LOCM had been suggested by initial studies, however, subsequent studies could not confirm these findings (19-21). A review of literature performed by Morcos et al in 2008 showed that only three studies and one metaanalysis had demonstrated a lesser risk of CIN with IOCM compared with LOCM. One study had shown a higher incidence of CIN following the use of IOCM (21). Higher viscosity of IOCM may account for the possible mechanism underlying their nephrotoxic potential. Since our study was an observational study, the choice of contrast agent was determined by clinical decisions in an individual patient rather than randomized double-blind allocation of the patients into IOCM or LOCM groups. Consequently, those who received IOCM had more risk factors associated with CIN than those who received LOCM. Also, the number of patients who received IOCM in our study was considerably smaller than those who received LOCM (4.05\% [IOCM] as compared to $95.95 \%$ [LOCM]). A large randomized double-blinded trial could establish stronger evidence regarding the incidence of CIN in these two groups.

Heart failure predisposes to increased risk of CIN. The possible underlying mechanism could be the decrease in effective circulatory volume, release of vasoconstrictor substances, inadequate hydration and failure to maintain adequate diuresis (22-24).

Serum creatinine is not an ideal predictor of renal function. But still it is the most commonly used marker for assessment of renal function and the definition of CIN in literature is mostly based on serum creatinine levels. In our study, we found that the baseline serum creatinine levels of those who developed CIN were higher than those who did not develop CIN $(1.32 \pm 0.33 \mathrm{mg} / \mathrm{dL}$ [CIN group] as compared to $0.91 \pm 0.24 \mathrm{mg} / \mathrm{dL}$ [no CIN group], $P<0.00001)$. The post-procedure serum creatinine level was also higher among those who developed CIN $(2.54 \pm 1.74 \mathrm{mg} / \mathrm{dL}$ [CIN group] as compared to $0.98 \pm 0.28$ $\mathrm{mg} / \mathrm{dL}$ [no CIN group], $P<0.00001)$. These results suggest that a higher pre-procedure serum creatinine level can predispose to the risk of CIN. Pre-existing renal insufficiency is the most important and predictive risk factor for CIN $(8,25,26)$.

Our data suggests that the various established prophylactic measures for prevention of CIN were not effective in our study population. The incidence of CIN among those who received preventive measures was found to be higher than those who did not. Since our study was an observational study, we could not randomize patients who received prophylaxis and those who did not. Consequently, the patients who received reno-protective measures had more associated confounding risk factors than those who did not receive any prophylactic measure prior to the procedure. Most of the patients who received prophylactic measures were inpatients and had a higher pre-procedure serum creatinine level. Another possible explanation for this observation could be that the preventive measures were not administered as per the recommended protocol and were inadequate or ineffective. A randomized controlled double-blinded trial could better evaluate the role of various preventive measures.

Our study involved many different variables and many confounding factors. To determine independent predictors for CIN in our study, we performed multivariate logistic regression using those variables which we found significant in univariate analysis.

The logistic regression model yielded heart failure $(P=0.001)$, pre-procedure serum creatinine $\geq 1.5 \mathrm{mg} /$ $\mathrm{dL} \quad(P=0.005)$ and nature of contrast material used $(P=0.001)$ as independent predictors of CIN in our study. The receiver operator characteristic (ROC) curve yielded an area under cure (AUC) of 0.9628 which indicates that our model had good discrimination and calibration characteristics. Validation group would be required to further evaluate these independent predictors of CIN. Most of the literature on CIN lacks comparison between those who receive contrast agent and those who do not. Those studies which have included a control group, including the studies by Cramer et al (27) and Heller et al (28), have demonstrated that the effect of contrast agents on serum creatinine levels was not significantly different from the variations in serum creatinine levels among those who do not receive any contrast agent. Our observations are significantly different from those of Cramer et al and Heller et al. It is important to consider that both these studies used a different definition for CIN than what is most commonly used in the literature. They considered CIN as an increase in serum creatinine levels of $\geq 50 \%$ from the baseline $(27,28)$. Since the threshold for diagnosis used by them was much higher than our study, it is possible that they may not have identified many cases of CIN which would otherwise be diagnosed as CIN had a lower threshold been used. Furthermore, the population characteristics in their study may have been very different from our study.

Five patients (17.85\%) who developed CIN required renal replacement therapy in the form of hemodialysis or 
peritoneal dialysis. All of these patients were inpatients. Four out of 28 patients (14.28\%) died after developing CIN and all of the patients were inpatients. Although, it is difficult to establish that CIN was actually the cause of the death in these patients, a reasonable inference which could be drawn from this data is that the development of CIN among hospitalized patients increases the risk of renal replacement therapy and in-hospital mortality.

\section{Conclusion}

In conclusion, CIN is an important complication of intravascular iodinated contrast administration and is associated with an increased risk of in-hospital renal replacement therapy and mortality. Heart failure, preprocedure serum creatinine level of $\geq 1.5 \mathrm{mg} / \mathrm{dL}$ and nature of contrast medium can independently predict the probability of CIN. Large double-blinded randomized controlled trial may further clarify the role of prophylactic measures in CIN and relative nephrotoxicities of isoosmolar contrast media as compared to low-osmolar contrast media.

\section{Limitations of the study}

Low proportion of patients is a limitation of our study. Observational nature of the study is also a limitation of the study. A randomized blinded clinical trial would better evaluate the epidemiology of CIN.

\section{Authors' contribution}

SP participated in the acquisition of the data. NAC and TAG prepared the research plan and co-ordinated the study design. SP participated in the analysis of the data and prepared the final manuscript. NAC and TAG approved the final manuscript.

\section{Conflicts of interest}

All the authors declare that they have no conflict of interest in this research.

\section{Ethical considerations}

Ethical issues (including plagiarism, data fabrication, double publication) have been completely observed by the authors.

\section{Funding/Support}

This article is extracted from the radiology thesis of Dr Sameer Peer. This study did not receive any financial support or any other support from government, private or commercial organization or any other source.

\section{References}

1. Shusterman N, Strom BL, Murray TG, Morrison G, West SL, Maislin G. Risk factors and outcome of hospital-acquired acute renal failure: clinical epidemiologic study. Am J Med 1987;83:65-71. doi: 10.1016/0002-9343(87)90498-0.

2. Nash K, Hafeez A, Hou S. Hospital-acquired renal insufficiency. Am J Kidney Dis 2002;39:930-6. doi: 10.1053/ ajkd.2002.32766.
3. Hou SH, Bushinsky DA, Wish JB, Cohen JJ, Harrington JT. Hospital-acquired renal insufficiency: a prospective study. Am J Med. 1983;74:243-8. doi: 10.1016/00029343(83)90619-8.

4. Mehran R, Nikolsky E. Contrast-induced nephropathy: definition, epidemiology and patients at risk. Kidney Int. 2006;100:S11-15. doi: 10.1038/sj.ki.5000368.

5. McCullough PA, Wolyn R, Rocher LL, Levin RN, O'Neill WW. Acute renal failure after coronary intervention: incidence, risk factors, and relationship to mortality. Am J Med. 1997;103:368-75. doi: 10.1016/S0002-9343(97)001502

6. Katzberg RW, Newhouse JH. Intravenous contrast mediuminduced nephrotoxicity: is the medical risk really as great as we have come to believe? Radiology. 2010;256:21-8. doi: 10.1148/radiol.10092000.

7. Persson PB, Hansell P, Liss P. Pathophysiology of contrast medium induced nephropathy. Kidney Int. 2005;68:14-22. doi: 10.1111/j.1523-1755.2005.00377.x.

8. Gleeson TG, Bulugahapitiya S. Contrast-induced nephropathy. AJR Am J Roentgenol. 2004;183:1673-89. doi: 10.2214/ajr.183.6.01831673.

9. Kolonko A, Kokot F, Wiecek A. Contrast-associated nephropathy-old clinical problem and new therapeutic perspectives. Nephrol Dial Transplant. 1998;13:803-6.

10. Rudnick MR, Kesselheim A, Goldfarb S. Contrast-induced nephropathy: how it develops, how to prevent it. Cleve Clin J Med. 2006;73:75-80.

11. Schweiger MJ, Chambers CE, Davidson CJ, Blankenship J, Bhalla NP, Block PC, et al. Prevention of contrast induced nephropathy: recommendations for the high risk patient undergoing cardiovascular procedures. Catheter Cardiovasc Interv. 2007;69:135-40. doi: 10.1002/ccd.20964.

12. McCullough PA, Wolyn R, Rocher LL, Levin RN, O'Neill WW. Acute renal failure after coronary intervention: incidence, risk factors, and relationship to mortality. Am J Med. 1997;103:368-75. doi: 10.1016/S0002-9343(97)001502

13. Weisbord SD, Mor MK, Resnick AL, Hartwig KC, Palevsky PM, Fine MJ. Incidence and outcomes of contrast-induced AKI following computed tomography. Clin J Am Soc Nephrol. 2008;3:1274-81. doi: 10.2215/CJN.01260308.

14. Kim SM, Cha RH, Lee JP, Kim DK, Oh KH, Joo KW, et al. Incidence and outcomes of contrast-induced nephropathy after computed tomography in patients with CKD: a quality improvement report. Am J Kidney Dis. 2010;55:1018-25. doi: 10.1053/j.ajkd.2009.10.057.

15. Mitchell AM, Jones AE, Tumlin JA, Kline JA. Incidence of contrast-induced nephropathy after contrast-enhanced computed tomography in the outpatient setting. Clin J Am Soc Nephrol. 2010;5:4-9. doi: 10.2215/CJN.05200709.

16. Mitchell AM, Kline JA. Contrast nephropathy following computed tomography angiography of the chest for pulmonary embolism in the emergency department. J Thromb Haemost. 2007;5:50-4. doi: 10.1111/j.15387836.2006.02251.x.

17. Chen SL, Zhang J, Yei F, Zhu Z, Liu Z, Lin S, et al. Clinical outcomes of contrast-induced nephropathy in patients undergoing percutaneous coronary intervention: a prospective, multicenter, randomized study to analyze the effect of hydration and acetylcysteine. Int J Cardiol. 2008; 126:407-13. doi: 10.1016/j.ijcard.2007.05.004.

18. Stacul F, van der Molen AJ, Reimer P, Webb JA, Thomsen HS, 
Morcos SK, et al. Contrast induced nephropathy: updated ESUR Contrast Media Safety Committee guidelines. Eur Radiol. 2011;21:2527-41. doi: 10.1007/s00330-011-2225-0.

19. Solomon R. The role of osmolality in the incidence of contrast-induced nephropathy: a systematic review of angiographic contrast media in high risk patients. Kidney Int. 2005;68:2256-63. doi: 10.1111/j.15231755.2005.00684.x.

20. Heinrich MC, Haberle L, Muller V, Bautz W, Uder M. Nephrotoxicity of iso-osmolar iodixanol compared with nonionic low-osmolar contrast media: meta-analysis of randomized controlled trials. Radiology. 2009;250:68-86. doi: $10.1148 /$ radiol.2501080833.

21. Morcos SK. Contrast-induced nephropathy: are there differences between low osmolar and iso-osmolar iodinated contrast media? Clin Radiol. 2009;64:468-72. doi: 10.1016/j. crad.2008.08.019.

22. Heyman SN, Reichman J, Brezis M. Pathophysiology of radiocontrast nephropathy: a role for medullary hypoxia. Invest Radiol. 1999;34:685-691.

23. Dangas G, Iakovou I, Nikolsky E, Aymong ED, Mintz GS, Kipshidze NN, et al. Contrast-induced nephropathy after percutaneous coronary interventions in relation to chronic kidney disease and hemodynamic variables. Am J Cardiol 2005;95:13-9. doi: 10.1016/j.amjcard.2004.08.056.

24. Stevens MA, McCullough PA, Tobin KJ, Speck JP, Westveer DC, Guido-Allen DA, et al. A prospective randomized trial of prevention measures in patients at high risk for contrast nephropathy: results of the P.R.I.N.C.E. study-Prevention of Radiocontrast Induced Nephropathy Clinical Evaluation. J Am Coll Cardiol. 1999;33:403-11. doi: 10.1016/S07351097(98)00574-9.

25. Segal AJ, Ellis JH, et al. ACR, Manual on Contrast Media: Version 6. American College of Radiology; 2008.

26. Keevil BG, Kilpatrick ES, Nichols SP, Maylor PW. Biological variation of cystatin C: implications for the assessment of glomerular filtration rate. Clin Chem. 1998;44:1535-9.

27. Cramer BC, Parfrey PS, Hutchinson TA, Baran D, Melanson $\mathrm{DM}$, Ethier RE, et al. Renal function following infusion of radiologic contrast material. Arch Intern Med. 1985;145:87-9.

28. Heller CA, Knapp J, Halliday J, O’Connell D, Heller RF. Failure to demonstrate contrast nephrotoxicity. Med J Aust. 1991;155:329-332.

Copyright $\odot 2017$ The Author(s); Published by Nickan Research Institute. This is an open-access article distributed under the terms of the Creative Commons Attribution License (http://creativecommons.org/licenses/by/4.0), which permits unrestricted use, distribution, and reproduction in any medium, provided the original work is properly cited. 